\title{
Individual differences in the mixture ratio of rotation and nonrotation trials during rotated mirror/normal letter discriminations
}

\author{
Jordan A. Searle • Jeff P. Hamm
}

Published online: 4 January 2012

(C) Psychonomic Society, Inc. 2011

\begin{abstract}
Mental rotation is thought to underlie the increase in response times (RTs) for deciding whether rotated letters are normal or mirrored versions. However, mental rotation predicts a linear increase in RTs, whereas the mirror/normal letter discrimination task typically produces a curved function. Recently, Kung and Hamm suggested that this curved function results from a mixture of trials in which mental rotation is employed and trials in which it is not. The mixture ratio may vary between individuals, with some individuals relying more on mental rotation than others. There is no factor in the Kung and Hamm model that reflects such individual differences. In the present study, we suggest that a possible exponent parameter could be added to the Kung and Hamm model to capture individual differences in the mixture ratio. This exponent parameter appears to capture an individual characteristic since the value obtained correlates between the mirror/normal letter task and a left/right object facing task. The development of a quantity that represents the mixture ratio will aid further testing of processes involved in the visual imagery system.
\end{abstract}

Keywords Imagery $\cdot$ Individual differences $\cdot$ Mental rotation

When deciding whether a rotated alphanumeric character is either normal or mirrored, it has generally been accepted that one has to imagine the character rotated back to the normal upright prior to making this decision. This imagined transformation of the character to a new orientation is

J. A. Searle · J. P. Hamm $(\bowtie)$

Research Centre for Cognitive Neuroscience,

Department of Psychology, City Campus,

The University of Auckland,

Private Bag 92019,

Auckland, New Zealand 1142

e-mail: j.hamm@auckland.ac.nz reflected in response times (RTs), which increase with further rotation away from the upright. The mental realignment process, referred to as mental rotation (Cooper \& Shepard, 1973; Shepard \& Metzler, 1971), is thought to reflect the holistic and analog nature of this mental imagery transformation.

Mental rotation, as found in mirror/normal discriminations of block figure pairs, produces a characteristic RT function. First, RTs tend to increase a constant amount for every degree of angular difference between the two items. Second, the RTs are maximal for $180^{\circ}$ of rotation, indicating that the rotation tends to proceed in the shortest angular direction required to align the two items - that is, clockwise or counter-clockwise for rotations in the picture plane. Finally, the rate of mental rotation appears to be roughly equal for depth and picture plane rotations (Shepard \& Metzler, 1971).

The linear increase in RT as a function of stimulus orientation is often considered the hallmark of mental rotation. However, there are tasks such as the top/bottom discrimination task that produce similar RT functions but for different reasons (Harris, Harris, \& Caine, 2002; Light \& Hamm, 2008). Indeed, discriminating between left and right hands is sometimes referred to as a mental rotation task (Amick, Schendan, Ganis, \& Cronin-Golomb, 2006; Thayer \& Johnson, 2006; Thayer, Johnson, Corballis, \& Hamm, 2001); however, it is probably more appropriate to refer to it as a mental movement task that is more influenced by motor imagery (Parsons, 1987) than by the visual imagery process of mental rotation. However, it is probably best not to refer to tasks as mental rotation tasks or motor imagery tasks but to restrict the use of these terms to the psychological processes they describe. It is quite possible for a task to involve multiple forms of imagery. We use the term mental rotation here to specifically refer to the visual imagery transformation.

Mental rotation has even been suggested to be employed to determine an object's orientation (De Caro \& Reeves, 
2000); however, if orientation information is not available prior to mental rotation, then how it could be possible to determine the shortest angular direction to the upright becomes logically problematic. Similarly, the theoretical notion of mental rotation prior to object identification (Jolicoeur, 1990) has generally been questioned not because of a failure of the empirical data to correspond to that predicted by mental rotation, but rather because of the difficulty in determining how the stimulus orientation could be determined if the stimulus has not already been identified, at least to some rudimentary level (Corballis, 1988; Hamm \& McMullen, 1998). Indeed, Cooper and Shepard (1973) originally assumed that letter identification occurred invariant of orientation and that mental rotation was only necessary to distinguish normal characters from their mirrored versions. It has been suggested that mental rotation is required only when the task requires the discrimination of mirror images (Corballis, 1982; Corballis \& McLaren, 1984). It is curious that discriminating mirrored and normal versions of rotated letters typically produces an RT function that deviates from the predicted linear increase (Cooper \& Shepard, 1973; Kung \& Hamm, 2010).

The deviation from linearity is thought to arise because mental rotation may not always be employed when the characters are near the upright (Cooper \& Shepard, 1973; Kung \& Hamm, 2010), so the overall RT at any orientation reflects a mixture of both rotation and nonrotation trials. Kung and Hamm suggested that this nonrotation process is based upon knowledge of the direction in which mental rotation is to proceed. They posited that when mental rotation is to proceed through a counter-clockwise direction (i.e., a stimulus at $60^{\circ}$ or $120^{\circ}$ clockwise), the top pole of the stimulus' vertical axis will move toward the left pole of the stimulus' horizontal axis. When the stimulus is to be mentally rotated clockwise, the top pole of the stimulus' vertical axis will move toward the right pole of the stimulus' horizontal axis. This directional information is thought to be enough to polarize the stimulus' horizontal axis and to determine which pole would be the left pole and which would be the right pole without actually performing the time-consuming mental rotation process. However, when a stimulus is inverted, the aforementioned nonrotation process codes a pole that is on the viewer's left as being the right pole, and a pole that is on the viewer's right as being the left pole. This spatial conflict is suggested to trigger mental rotation so that the conflict can be resolved. It is presumed that there is a gradient of this conflict between upright stimuli and inverted stimuli, so because the conflict is thought to increase with stimulus orientation (Light \& Hamm, 2008), this results in mental rotation being triggered more frequently, and in the nonrotation process being relied upon less frequently, as a function of stimulus orientation.

Furthermore, Kung and Hamm (2010) proposed a model of rotated mirror/normal letter discriminations in which they quantified the mixture between mental rotation and nonrotation trials and suggested that the proportion of trials that require mental rotation at any given orientation is equal to the angle, in degrees from upright, over 180 . This means that characters at $60^{\circ}$ are rotated $60 / 180$, or on one-third of trials, whereas those at $120^{\circ}$ are rotated on two-thirds of trials, and so forth.

In addition to the mixture of rotation and nonrotation trials, there is evidence to suggest that discriminating mirror alphanumeric characters from normal ones involves an additional out-of-plane "flip" of mirror stimuli (Hamm, Johnson, \& Corballis, 2004; Kung \& Hamm, 2010). This theory of an additional rotation for mirror stimuli is based upon electrophysiological data (EEG; Hamm et al., 2004) and also upon a positive relationship between an individual's rate of rotation and the RT difference they show between the mirror and normal conditions (Hamm et al., 2004; Kung \& Hamm, 2010).

Kung and Hamm (2010) demonstrated that RT functions, at both the group and individual level, could be predicted using only RTs for the upright and inverted conditions, collapsed over mirror and normal conditions. They suggested, ignoring the mirror and normal difference for now, that:

$\mathrm{Rt}_{\theta}=\theta / 180 * \mathrm{Rt}_{\text {rotation }}+[1-(\theta / 180)] * \mathrm{Rt}_{0}$,

where $\mathrm{Rt}_{\theta}$ is the $\mathrm{RT}$ at a given orientation $(\theta) . \mathrm{Rt}_{\text {rotation }}$ is the estimated RT based upon the linear interpellation of RTs between upright and inverted. The terms $\theta / 180$ and $[1-(\theta /$ 180)] reflect the proportional mixture of trials in which mental rotation is employed and the remaining, nonrotation trials (with the presumed RT equal to that observed for upright trials). $\mathrm{Rt}_{0}$ represents the $\mathrm{RT}$ for upright trials, or orientation of $0^{\circ}$. In the equation that follows, $\mathrm{Rt}_{180}$ represents the $\mathrm{RT}$ for inverted trials, or orientation of $180^{\circ}$.

Because $\mathrm{Rt}_{\text {rotation }}$ is equivalent to $\theta\left(\mathrm{Rt}_{180}-\mathrm{Rt}_{0}\right) / 180+$ $\mathrm{Rt}_{0}$, the previous equation can be rearranged and expressed as follows:

$\mathrm{Rt}_{\theta}=\mathrm{Rt}_{0}+\theta / 180 * \mathrm{Rt}_{\text {rotation }}$

This relationship captures the curved shape of the RT function. It also demonstrates that the RT pattern can be described by a function based upon an orientation-related mixture of mental rotation and nonrotation trials. The $\mathrm{Rt}_{\text {rotation }}$ term captures an individual's orientation effect magnitude (ms/degrees, or the inverse of their rate of rotation, hereafter referred to as simply the orientation effect), derived from the observed data $\left(\mathrm{Rt}_{180}-\mathrm{Rt}_{0}\right) / 180$. With the difference in RTs between mirror and normal trials also being derived from an individual's orientation effect (Hamm et al., 2004; Kung \& Hamm, 2010), it becomes apparent that the entire RT function can be calculated from having only the mean RT for upright and inverted stimuli (Kung \& Hamm, 2010). Effectively, Eq. 2 models the RT function according to two theoretical psychological parameters: first, an individual's orientation 
effect, and second, an individual's baseline RT. The former is determined by the difference in RTs between upright and inverted stimuli and is converted to reflect milliseconds per degree of orientation from the upright. The latter is estimated by the RT for upright stimuli collapsed over the mirror and normal conditions. The shape, or curve, of the function is determined by the $\theta / 180$ term, which is a function of the stimulus orientation and is independent of the participant. This stimulus condition term is what captures the mixture ratio between mental rotation trials and nonrotation trials. Because this mixture term does not involve the participant, the implication is that this ratio is relatively constant across individuals.

However, Kung and Hamm (2010) noted that some participants appeared to show less curvature than others, suggesting that individual differences may exist with respect to the mixture ratio. As described previously, the recent mixture model does not include a parameter that would capture such individual differences. The first objective of the present investigation was to determine whether the mixture model of Kung and Hamm can be modified to include a parameter that captures individual variation in the proportional mix between mental rotation trials and nonrotation trials. This parameter would have to operate on the mixture term, namely the $\theta / 180$ proportion. In addition, for individuals who always employ mental rotation, as Kung and Hamm suggested at least four of their participants may, then this parameter should convert the $\theta / 180$ term to 1.0 at all orientations.

It is here suggested that an exponent may be required for this term of the model $(\theta / 180)^{\mathbf{X}} * \mathrm{Rt}_{\text {rotation. }}$. For individuals who always employ mental rotation, the exponent would be equal to 0 [i.e., $(\theta / 180)^{0} * \mathrm{Rt}_{\text {rotation }}$ ] and for those who were more prone to respond without rotation, this value would increase. According to the group data presented in Kung and Hamm (2010), the exponent for group grand average data appears to be approximately 1.0 .

Kung and Hamm (2010) indicated that the left/right facing task is highly similar to the mirror/normal letter discrimination task except that the stimuli employed are typically common objects. These objects are generally depicted in a roughly profile view, so when they are shown upright, they are clearly facing either to the left or the right. In contrast with the difference in RTs typically found for mirror and normal characters, RTs for left- and right-facing objects are similar, which suggests that the proposed flip of mirror stimuli (Hamm et al., 2004; Kung \& Hamm, 2010) does not occur in this task.

Although mental rotation appears to be consistently employed when discriminating items that are horizontally symmetric (i.e., $p$ from $b$ or $q$ from $d$ ), it appears that discriminating vertically symmetric pairs (i.e., $p$ from $q$ or b from d) is more prone to this mixture of rotation and nonrotation trials (Corballis \& McLaren, 1984; Kung \& Hamm, 2010). The literature does mention that the RT curves for discrimination of left- and right-facing objects show an acceleration type profile (Corballis \& McLaren, 1984; Jolicoeur, 1988; Light \& Hamm, 2008; McMullen \& Jolicoeur, 1990); therefore, this task may also employ a mixture of rotation and nonrotation trials. Moreover, if the left/right facing task and the mirror/normal discrimination task both employ the common underlying mental process of mental rotation, then the orientation effect should be of similar magnitude between the tasks. In addition, the estimated orientation effects for individuals should correlate between these tasks. If, as suggested by the literature, the left/right facing task produces a curved RT function, then the exponent terms for individuals should also correlate between the tasks. Finally, the left/right facing task is known to produce asymmetric RT functions, with left-facing objects responded to more quickly when rotated $60^{\circ}$ or $120^{\circ}$ clockwise than counter-clockwise, and with rightfacing objects showing the opposite pattern. Because the letters, F, G, L, P, and R (employed during mirror/normal letter discriminations) all face to the right (or at least in a common direction), whereas $\mathrm{J}$ could be viewed to face to the left (or in the opposite direction), then it is possible for a similar interaction to occur. Consistent with this notion, Kung and Hamm (2010) did report an interaction between facing and the comparison between $30^{\circ}$ and $330^{\circ}$. If, therefore, this asymmetry is also an individual characteristic, then the asymmetry in the RTs should correlate between tasks. This asymmetry, however, is not currently modeled in the mixture model, nor is it the major focus of the present investigation.

\section{Method}

\section{Participants}

Thirty-four participants volunteered in this study, and all reported normal or corrected-to-normal vision. Data gained from 10 participants were not used for analysis because they failed to meet the inclusion criteria of a mean accuracy across both tasks of at least $85 \%$, as well as of mean accuracies of at least $65 \%$ for responses to mirror or normal letter, or to left- or right-facing objects, at all of the six orientations.

The twenty-four remaining participants (12 male, 12 female) ranged in age from 20 to 34 years old (mean age $=22.71, S D=2.85$ ). All participants were right-handed, as assessed on the Edinburgh Handedness Inventory (laterality quotient range 43 to 100; Oldfield, 1971). All procedures were approved by the University of Auckland Human Participants Ethics Committee.

\section{Stimuli}

The mirror/normal letter task consisted of six different uppercase letter stimuli (taken from Kung \& Hamm, 2010; see 
Fig. 1a). Each letter was presented 12 times in its normal version and 12 times in its mirror version at each orientation $\left(0^{\circ}, 60^{\circ}, 120^{\circ}, 180^{\circ}, 240^{\circ}\right.$, and $300^{\circ}$; see Fig. $1 \mathrm{~b}$ and c $)$.

The left/right facing task consisted of six different line drawings of objects (taken from the set employed in Hamm \& McMullen, 1998; see Fig. 1d). Each object was presented 12 times facing left and 12 times facing right for each orientation $\left(0^{\circ}, 60^{\circ}, 120^{\circ}, 180^{\circ}, 240^{\circ}\right.$, and $300^{\circ}$; see Fig. 1e and f). For both objects and letters, the major axis was between 4.2 and 4.4 degrees of visual angle. The major axis was the vertical for the letters, and the horizontal for the objects. The heights of the objects varied between 2.6 and 4.2 degrees, and the widths of the letters were between 2.2 and 2.7 degrees.

Stimuli were presented on a color computer monitor with a screen resolution of $800 \times 600$ pixels. Image presentation and timing of responses started with the screen refresh cycle (Heathcote, 1988) and then synchronized with the uppermost pixel of the stimulus, using the timing routine as recommended by Hamm (2001). Participants used a chinrest to keep their eyes $57 \mathrm{~cm}$ from the center of the screen.
Procedure

Each task consisted of two blocks of 432 trials. Half of the participants responded using their right hands for the first block of trials and their left hands for the second block, with the hand order reversed for the two blocks of the second task. Hand order was reversed for the remaining participants. Individuals were given a break between each block. Individuals completed both the mirror/normal letter task and the left/right facing task, with half of the individuals completing the mirror/normal letter task first and the other half completing the left/right facing task first, ensuring that hand order was crossed with task order.

Mirror/normal letter task Individuals performed 432 mirror and 432 normal letter discriminations. Responses to mirror letters were made by pressing the " 1 " key on a number pad, whereas responses to normal letters were made by pressing the "2" key. Each letter remained on the screen until the individual responded or until 4,000 ms had passed. Trials were presented in a randomized order.
Fig. 1 a Letter stimuli used in the mirror/normal letter task presented in normal versions at $0^{\circ}$. b An example presented at each of six orientations in its normal version and $\mathbf{c}$ in its mirror version. d Object stimuli used in the left/right facing object task presented in leftfacing versions at $0^{\circ}$. e An example presented at each of six orientations in its left-facing version and $\mathbf{f}$ in its right-facing version
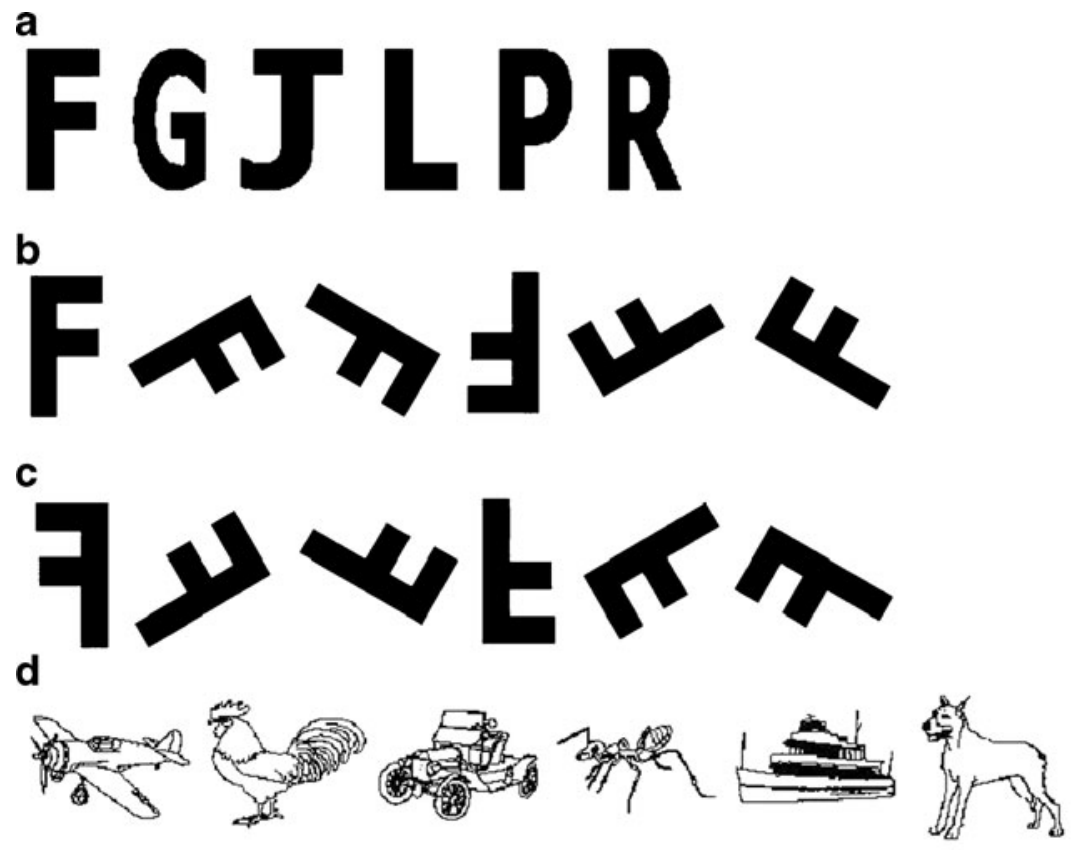

e
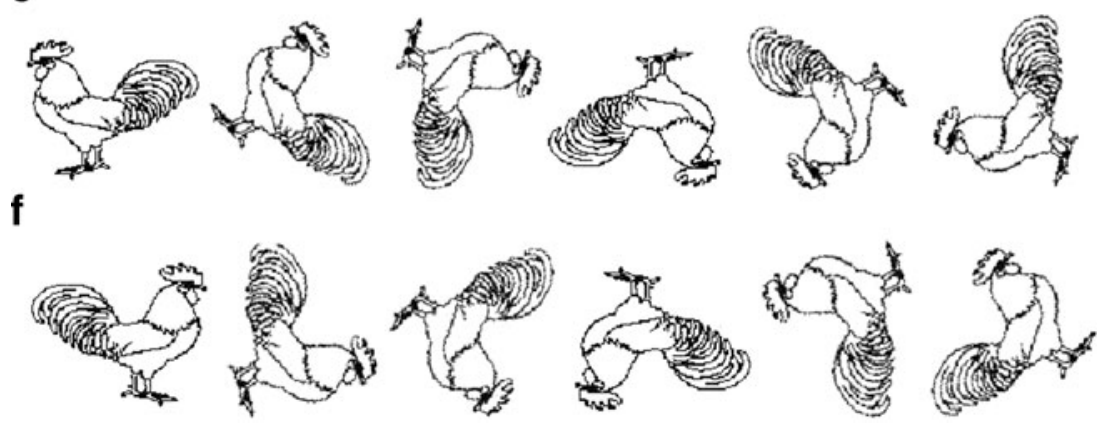
Left/right facing task Individuals each performed 432 leftand 432 right-facing object discriminations. Responses to object facing were made similarly to responses in the mirror/ normal letter task, with responses to left-facing objects being made by pressing the " 1 " key and responses to right-facing objects being made by pressing the "2" key. Each object remained on the screen until the individual responded or until 4,000 ms had passed. Trials were presented in a randomized order.

\section{Results}

In all figures, the data shown for stimuli presented at $360^{\circ}$ is the same data as shown for $0^{\circ}$. They are included to assist the visual assessment of symmetry and are included only once in the statistical analysis. All orientations refer to clockwise stimulus rotations.

The mean RTs for correct responses in the mirror/normal letter and the left/right facing task, separated by their respective responses, can be seen below in Fig. 2a and b, respectively. Figure 2c shows the mean RTs collapsed over responses for comparison between the tasks. Figure $2 \mathrm{~d}-\mathrm{f}$ show the corresponding plots of response accuracy. Individual response accuracies were negatively correlated with individual RTs, indicating that the results are not due to a speed/accuracy trade-off, $r(574)=-0.48, p<.05$. Note that this negative correlation holds if the correlations are computed for the tasks separately, $r(286)=-0.36$ and $-0.51, p<$ .05 (for mirror/normal and left/right, respectively), or if the correlations are simply performed on the group mean data, $r(10)=-0.96$ and $-0.94, p<.05$ (for mirror/normal and left/ right, respectively).

Calculation of parameters for individuals

It is being suggested that individual participants' RTs in the mirror/normal letter task may be modeled by the equation:

$\mathrm{Rt}_{\theta}=\mathrm{Rt}_{0}+(\theta / 180)^{\mathrm{X}} *\left[\theta\left(\mathrm{Rt}_{180}-\mathrm{Rt}_{0}\right) / 180\right]$,

where $\theta\left(\mathrm{Rt}_{180}-\mathrm{Rt}_{0}\right) / 180$ is the change in $\mathrm{RT}$ that one would obtain if mental rotation produced a linear increase on RTs, $\mathrm{Rt}_{0}$ is the baseline $\mathrm{RT}$ for upright stimuli (collapsed over mirror and normal trials), $\mathrm{Rt}_{180}$ is the $\mathrm{RT}$ for inverted stimuli (again collapsed over mirror and normal trials), $\theta / 180$ is the mixture ratio weighting that effectively reduces the influence
Fig. 2 Mean observed response times as a function of orientation for the a mirror/ normal letter task, $\mathbf{b}$ left/right facing task, and $\mathbf{c}$ both tasks collapsed over response options. Mean observed accuracies as a function of orientation for the $\mathbf{d}$ mirror/ normal letter task, e left/right facing task, and $\mathbf{f}$ both tasks collapsed over response options
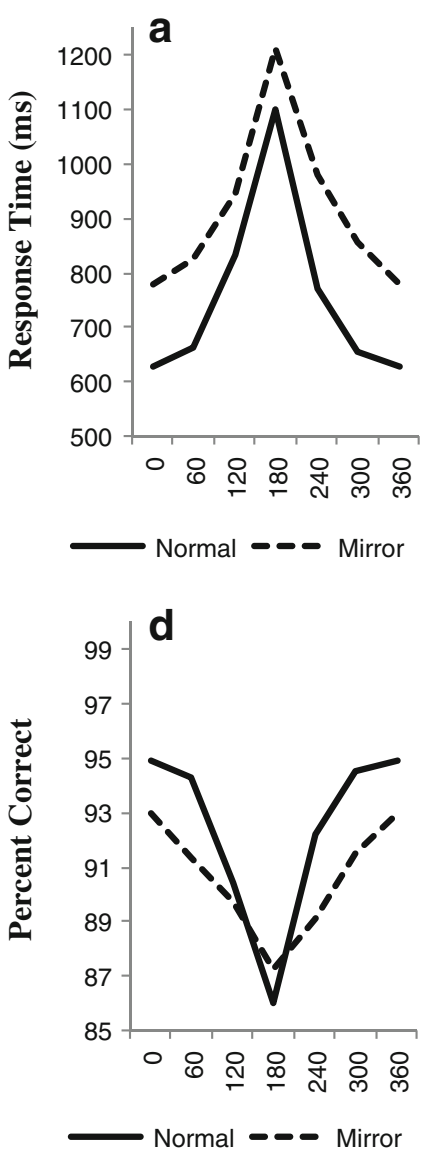
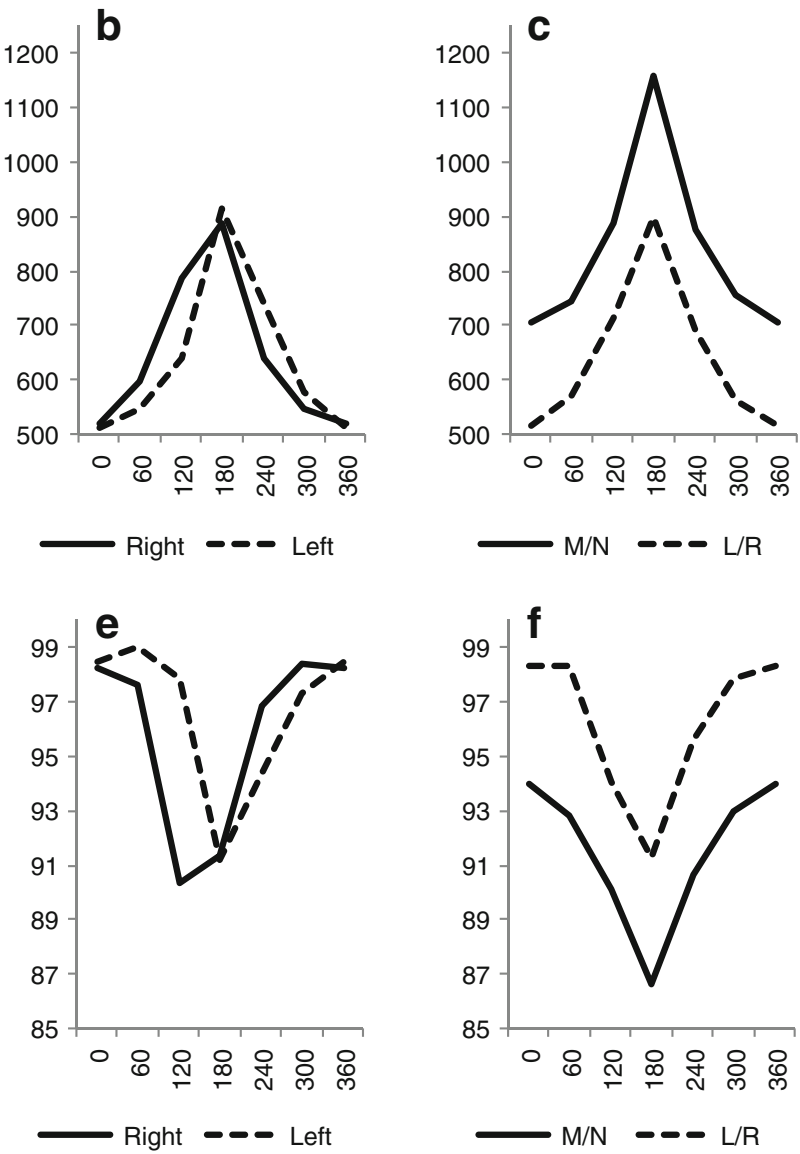

Orientation 
of mental rotation trials as a function of the stimulus orientation, and the exponent value is an individual parameter that increases or decreases the influence of this mixture. The term $\left(\mathrm{Rt}_{180}-\mathrm{Rt}_{0}\right) / 180$ quantifies an individual's orientation effect as the inverse of their rate of rotation.

The RT estimate from Eq. 3 is then adjusted to account for the difference in RTs between mirror and normal stimuli. The adjustment is based upon the individuals' orientation effect (Kung \& Hamm, 2010), so this adjustment does not require any additional participant parameters.

It is proposed that an individual's RT function, therefore, can be modeled by knowing three values for each participant. These three parameters are (a) their baseline RT, (b) their orientation effect, and (c) their mixture exponent. These parameters may also be calculated for the left/right facing task. As is typical of the mirror/normal letter task, RTs were, on average, slower to mirror trials $(933 \mathrm{~ms})$ than to normal trials $(775 \mathrm{~ms})$ when collapsed over orientation, $t(23)=9.19, p<.05$. It should be noted that the left/right facing task did not show the same main effect of response, $t(23)=0.85, p>.05(M \mathrm{~s}=656$ versus $662 \mathrm{~ms}$, left vs. right, respectively). Subsequently, an individual's mirror/normal difference $\left(\mathrm{MN}_{\mathrm{diff}}\right)$ for the mirror/normal letter task was also calculated. It was found that the mirror/normal difference was related to an individual's orientation effect, $r(22)=.57$, $p<.05$, replicating previous studies (Hamm et al., 2004; Kung \& Hamm, 2010). For the present data, the regression equation is: [OE is the individual participant's orientation effect, or $\left.\left(\mathrm{Rt}_{180}-\mathrm{Rt}_{0}\right) / 180\right]$

$\mathrm{MN}_{\text {diff }}=46.43 * \mathrm{OE}+40.87$.

An individual participant's baseline RT and orientation effect are estimated from the observed RTs. The former is simply the mean RT for upright stimuli, and the latter is the difference between inverted and upright stimuli, divided by $180^{\circ}$, to give a value in terms of $\mathrm{ms} / \mathrm{deg}$. To obtain an individual's exponent $(\mathrm{X})$ value, the value of the exponent value in Eq. 3 was adjusted to minimize the squared error between the modeled RTs and the participant's observed RTs collapsed over response (either mirror/normal or left/ right). Figure 3 shows the sum of squared error as a function of the exponent value for Participant 1.

Influence of task order

The influence of task order was examined, and for the left/ right facing task, none of the measures (baseline RT, orientation effect, and exponent value) differed between those who performed the task first and those who performed it following the mirror/normal letter task [all $t \mathrm{~s}(22)<1.0, p>$ $.05]$. However, for the mirror/normal letter task the exponent values were larger, $t(22)=2.67, p<.05(1.98$ vs. 1.13$)$, and the baseline RTs were faster, $\mathrm{t}(22)=3.16, p<.05$ (632 vs. 776)

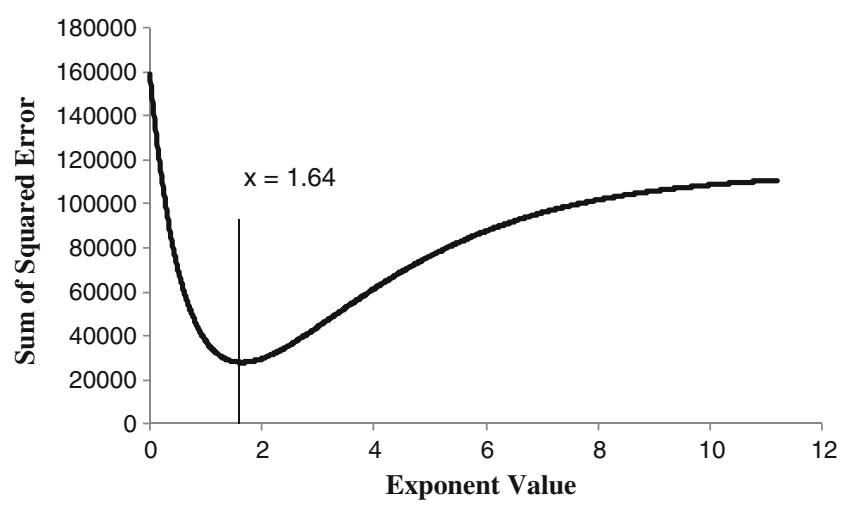

Fig. 3 Sum of squared error between modeled data and $X$ values for Participant 1's mirror/normal letter task response times

for those who performed the mirror/normal letter task after the left/right facing task than for those who performed the mirror/normal letter task first. The orientation effect for the mirror/normal letter task did not differ according to task order, $t(22)=1.53, p>.05$ (2.83 vs. 2.20 , performed first vs. second, respectively). However, one participant who performed the mirror/normal letter task second was an outlier with respect to their orientation effect, which was $3.5 \mathrm{SDs}$ above the mean. When they were dropped from the analysis, results indicated that, on average, the orientation effect was smaller for those who performed the mirror/normal letter task after the left/right facing task (1.84) than for those who performed it first $(2.83), t(21)=4.66, p<.05)$. The same pattern was found when using the nonparametric MannWhitney $\mathrm{U}$ test, with baseline RT and orientation effect smaller and exponent value larger for the mirror/normal letter task when it was performed after the left/right facing task than before (all $p \mathrm{~s}<.01$ ), whereas the parameters for the left/right facing task remained unchanged based upon task order (all $p \mathrm{~s}>.25$ ). Note, the difference in the orientation effect for the mirror/normal letter task was significant with the nonparametric test, with or without the inclusion of the outlier participant.

\section{Relationships between parameters}

To determine whether the parameters between the two tasks are related, correlations for the baseline RT, orientation effect, and exponent values were computed between the tasks. In order to reduce the effect of task order, the two groups defined by task order were separated, and each parameter was ranked from 1 to 12 for each task within their respective groups. These two series of ranks were then recombined, and a Spearman's rank order correlation was performed. This resulted in a significant correlation between the tasks for the baseline $\mathrm{RT}, r(22)=.56, p<.05$, orientation effect, $r(22)=.51, p<.05$, and for the exponent values, $r(22)=.42, p<.05)$. 
Correlations were also calculated on the aforementioned ranks between an individual's orientation effect and exponent value within the mirror/normal and the left/right facing task, separately. Neither of these comparisons was significant $[r(22)=-.05$, and .33, $p>.05$, for mirror/normal and left/right, respectively]. There was a significant correlation between the orientation effect ranking and baseline RT ranking for the mirror/normal task, $r(22)=.52, p<.05$, but not for the left/right task, $r(22)=.11, p>.05$. Finally, the baseline RT ranks did not correlate with the exponent value ranks for either task $[r(22)=-0.24$ and $-0.22(p>.05)$ for mirror/normal and left/right, respectively).

\section{Asymmetry of the response time functions}

The asymmetry of the RT functions for mirror letters and left-facing objects is quantified as the difference in the mean RTs between counter-clockwise rotations and clockwise rotations. The asymmetry for the normal and right-facing objects was quantified as the mean difference between clockwise and counter-clockwise rotations. Individual task asymmetry values were calculated by averaging the left and right asymmetries for the left/right facing task and the mirror/normal asymmetries for the mirror/normal letter task. ${ }^{1}$ On average, the left/right facing task showed larger asymmetry values $(82.7 \mathrm{~ms})$ than did the mirror/normal letter task $[33.7 \mathrm{~ms} ; t(23)=4.04, p<.05]$. However unlike the other parameters, these asymmetry values did not differ between task order for either task [33.8 vs. 33.5 for mirror/normal run first vs. second, respectively; 83.0 and 82.3 for left/ right run first vs. second, respectively; both $t \mathrm{~s}(22)<$ $0.03, p>.05]$. Finally, at the individual level, the asymmetry values did not correlate between tasks, $r(22)=.27, p>.05$, nor did the ranks correlate between tasks, $r(22)=0.27, p>.05$, even when the ranks were calculated to remove any effect of task order, $r(22)=0.29, p>.05$.

Is the increased model complexity justified?

There are three potential models under consideration. With respect to the change in RTs over orientation, the first model is that the RTs are a purely linear function of stimulus orientation. The second model is that proposed by Kung and Hamm (2010), with the orientation effect being a mix between the baseline RT and the linear increase RT, and with the mixture being a proportion between stimulus orientation

\footnotetext{
${ }^{1}$ Note, the asymmetry value for $\mathrm{J}(48.9)$ was similar to the asymmetry value as calculated for the "right-facing" letters (34.6) and did not show the sign reversal that would be expected if $\mathrm{J}$ should be considered a "left-" facing letter with respect to the asymmetry calculation.
}

and 180 that is constant between individuals. The third model, as proposed in the present article, varies the mixture for each participant by including an exponent term. With 12 orientations and 24 participants, this would result in 288 data points for the mirror/normal letter task, and 288 data points for the left/right facing task.

For the left/right facing task, the linear model contains 24 baseline RTs (one per participant) and 24 orientation effects, resulting in 48 parameters for the individual participants. In addition, there is the orientation of the stimulus (an additional parameter) and there is the residual sum of squares required for model evaluation (Burnham \& Anderson, 2002), resulting in a total of 50 parameters. Kung and Hamm's (2010) constant mixture model, using $\theta / 180$ as a constant mixture ratio, has the mixture ratio term as an additional parameter, for 51 parameters. Finally, the proposed model, which includes an individual mixture ratio for each participant by applying an exponent value to the mixture term, requires an additional 24 parameters (one exponent value per participant), for a total of 75 parameters.

The mirror/normal letter task produces three similar models, but each of these also includes the regression equation that accounts for the mirror/normal difference, so this requires inclusion of the stimulus condition (mirror/normal) and the two parameters of the regression equation (the slope and intercept). Therefore, parameters are added, resulting in models with 53, 54, and 78 parameters for the pure linear model, constant mixture model, and individual mixture model, respectively.

The models were compared using the Akaike criterion (Burnham \& Anderson, 2002). This comparison is performed by calculating the following term $\mathrm{ACC}_{\mathrm{c}}=$ $2 \mathrm{k}^{*}[\mathrm{n} /(\mathrm{n}-\mathrm{k}-1)]+\mathrm{n}[\ln (\mathrm{RSS} / \mathrm{N})]$, where $\mathrm{k}$ is the number of parameters in the model (as above), $\mathrm{n}$ is the number of data points (288 per task), and RSS is the residual sum of squares (Burnham \& Anderson, 2002; p. 66). The term $[\mathrm{n} /(\mathrm{n}-\mathrm{k}-1)]$ is used to inflate the penalty for increasing the number of parameters when the number of data points is less than approximately 40 times the number of parameters, as in the present situation. The preferred model is the one that produces the lowest $\mathrm{ACC}_{\mathrm{c}}$ value.

For both tasks, the most preferred model was the constant mixture model, followed by the individual mixture model, and the purely linear model ranked least preferred $\left(\mathrm{ACC}_{\mathrm{c}}\right.$ values of 2564.62, 2570.20, and 2734.85, for the left/right facing task, and 2609.72, 2625.82, and 2895.46 for the mirror/normal letter task). $\mathrm{ACC}_{\mathrm{c}}$ values are often expressed as a difference from the preferred model, resulting in the values of $[0: 5.58: 170.23]$ for the left/right facing task and $[0: 16.09: 285.74]$ for the mirror/normal letter task, for the constant mixture model (the preferred), individual mixture model $\left(2^{\text {nd }}\right)$, and pure linear model (least preferred). These 
translate to the constant mixture model's being preferred in the order of 16:1 over the individual mixture model for the left/right task, and over 3000:1 for the mirror/ normal task (Burnham \& Anderson, 2002; Wagenmakers \& Farrell, 2004).

In both cases, the individual mixture model would be the preferred model if it were not for the correction facture due to the low number of data points relative to the number of parameters, suggesting the aforementioned model selection may be based upon insufficient evidence. As such, baseline values, orientation effects, and individual exponent values were calculated for the mirror/normal letter task by drawing upon the data from two previously published studies (Hamm et al., 2004; Kung \& Hamm, 2010), including the four sets of behavioral data from the Hamm et al. study that were excluded because of EEG technical issues (which were not an issue for the present purpose), and four additional sets of data that were collected as pilots. This resulted in behavioral data sets from 68 different participants. Because the Kung and Hamm study employed orientations at $30^{\circ}$ increments rather than $60^{\circ}$, there were 24 data points from each of the 24 participants from this study, whereas the remaining 44 participants contributed 12 data points each. With the additional participants, the number of parameters for the mirror/ normal letter task models increased to 141,142 , and 210 (for linear, constant mixture, and individual mixture, respectively) and the number of data points was 1,104 . Note that the regression equation relating the orientation effects to mirror/normal difference was calculated over this entire data set and employed $\left[\mathrm{MN}_{\text {Diff }}=42.21 \mathrm{OE}+51.16 ; r(66)=.58\right.$, $p<.05]$. To use individual regression equations for each experiment would increase the number of parameters in each model even further, which runs counter to the purpose of attempting to build an explanatory model. It is presumed that basing the regression equation upon the largest data set will produce the most generalizable, and therefore useful, model. This resulted in $\mathrm{ACC}_{\mathrm{c}}$ values of 10827.44, 9793.82, and 9608.83, for the linear model, constant mixture model, and individual mixture model, respectively. These results indicate that, with the increased data included in the evaluation, the added complexity of the individual mixture model is to be preferred over the other two (difference from the preferred being $1218.61: 184.99: 0$ ). This translates to the individual difference model being preferred over the constant mixture model at an odds ratio $1.48 \mathrm{e}^{40}: 1$ (Burnham \& Anderson, 2002; Wagenmakers \& Farrell, 2004).

The extended data were used to compare the individual mixture model, in which individual parameters were employed for the baseline RT, the orientation effect, and the mixture exponent, against the seven simpler models that could be derived by replacing one or more of these parameters with the grand average value. For example, by setting all three parameters to the grand average values, one is testing whether any individual differences are justified or whether this nine-parameter model ${ }^{2}$ that employs the group grand averages will suffice. The individual mixture model was the most preferred of all simpler models. Note, the Kung and Hamm (2010) constant mixture model is also a model in which individual differences are assumed for the orientation magnitude and the baseline $\mathrm{RT}$, with the $\mathrm{X}$ value set at a constant value of 1 .

Similar comparisons were made for the left/right facing task. In this case, the most preferred model continued to be one that allowed for individual differences in the baseline $\mathrm{RT}$ and the orientation magnitude measures while maintaining a constant mixture exponent of 0.91 . The next most preferred model was the full individual differences model, which differed from the most preferred model by 5.81, which results in a preference ratio of approximately 18:1 (Burnham \& Anderson, 2002; Wagenmakers \& Farrell, 2004). It is presumed that, similar to the mirror/normal task, if a larger set of data were available, the full individual differences model would likewise be preferred.

\section{Comparison with literature}

It should be noted that the average exponent value of the participants is not the same value as the exponent value that is obtained when fitting the group average data. However, as an example demonstrating how past data could be examined with respect to these three parameters (baseline RT, orientation effect, and exponent values), the data from Rogers et al. (2002) will be used. RTs for the mirror/normal letter task were extracted from their Fig. 1 for their three groups of participants, which were a sample of healthy controls $(n=10)$, a sample of people diagnosed with nonmelancholic unipolar depression $(n=9)$, and a sample of people diagnosed with melancholic unipolar depression $(n=8)$. The groups' mean RTs were then fitted with parameters, as shown in Table 1.

In the Rogers et al. (2002) article, the results indicated that although both the melancholic and nonmelancholic groups showed generalized slowing overall, only the mel-

\footnotetext{
${ }^{2}$ The nine parameters in this model would be the stimulus orientation (a), the slope (b), and intercept (c) from the equation that relates the mirror/normal difference to orientation effect, which is used to derive the size of the mirror normal difference, the mirror/normal condition ( $d$; to decide whether the previous value should be added or subtracted), the mixture ratio constant (e; which is orientation/180), the group average orientation effect (f), the group average baseline response time $(\mathrm{g})$, the group average mixture exponent $(\mathrm{h})$, and the residual sum of squares (i) required to test the model.
} 
Table 1 Fitted parameters for Rogers et al.'s (2002) data

Baseline Response Orientation Effect $\mathrm{X}$

Time (in milliseconds) (ms/deg)

\begin{tabular}{llll}
\hline Controls (10) & 690.91 & 2.34 & 1.09 \\
Nonmelancholic (9) & 952.27 & 2.34 & 1.12 \\
Melancholic (8) & 1077.27 & 4.92 & 0.58 \\
\hline
\end{tabular}

ancholic group showed an increase in magnitude of orientation effect, indicating a deficit in mental rotation for this group. The exponent value, or mixture ratio, was not a known parameter at that time, so it could not have been tested.

Using the data from the 68 participants described previously, 10,000 groups of sample sizes 10, 9, and 8 were then generated by randomly selecting participants with replacement. Baseline RTs, orientation effects, and exponent values were then found for each of these random groups. This provided a distribution of these parameters according to groups of appropriate sample sizes. For all three parameters, the values for the controls from the Rogers et al. (2002) article were well within these distributions, at the 36.7, 39.3, and 29.5 percentile for the baseline RT, orientation effect, and exponent value, respectively. The nonmelancholic group showed a baseline RT that was slower than the entire sample of 10,000 groups of sample size 9, but their orientation effect and exponent values were well within their respective distributions (40.6 and 34.5 percentile, respectively). This replicates the finding of a "generalized slowing" with no influence on their magnitude of orientation effect, and extends this finding to no influence on their mixture proportions as well. The melancholic group also showed a baseline RT that was slower than the entire distribution of sample size 8 baseline RTs. Similarly, their orientation effect was larger than the entire distribution of sample size 8 orientation effects. These two findings replicate the results of the analysis in the Rogers et al. article. In addition, the exponent value fell at the 1.02 percentile, which would be considered significantly different at $p<.05$ in a two-tailed test, and would suggest that the melancholic depressed patients resorted to mental rotation more frequently than the control sample.

\section{Discussion}

Individual participants' RT functions for rotated mirror/ normal letter discriminations and rotated left/right facing object discriminations were modeled according to three separate parameters for each task. The three parameters were a baseline RT, a magnitude of their orientation effect (the inverse of their rate of rotation), and an exponent value that influenced the percentage of trials that are estimated to undergo mental rotation at any given orientation. With these three parameters, individual participants' RTs were modeled for each task (see Figures 4-11 in Appendix A and B for complete presentations of the individual models and RT data). The mirror/normal letter discrimination task also requires modeling of the overall difference in RT between mirror and normal stimuli, with this difference being derived from an individual's orientation effect. The calculated RTs captured, on average, $87 \%$ and $84 \%$ of the variance in a participant's RTs.

The three parameters were shown to correlate between the tasks, suggesting that all three measures were capturing something similar about an individual's performance, hence making them potentially useful measures of individual differences. In addition, the orientation effect measure and the mixture measure (the exponent), were not correlated with each other within either task, indicating that these measures were capturing something different about an individual's performance. Finally, although the orientation effect was correlated with the baseline measure for the mirror/normal letter task, this relationship was not found in the left/right facing task. This finding could reflect the fact that the baseline measure may, in part, reflect the mirror/normal difference because the baseline measure is the average of the upright normal and upright mirror trials, and the mirror/ normal difference was shown to be a function of the individual's orientation effect (Hamm et al., 2004; Kung \& Hamm, 2010). However, the orientation effect was also correlated with the upright normal RT, $r(22)=.49, p<$ .05 , a finding that did not replicate with the left/right facing task (using either left or right upright as "normal upright"). This effect was also not found when previously examined (Hamm et al., 2004), nor was it found in the data set from Kung and Hamm. In summary, the three parameters of baseline RT, orientation effect, and mixture exponent appear to be valid measures that reflect individual performance and which translate to other tasks and stimuli.

It should be noted that the asymmetry in the orientation functions, which is not captured by the present parameters, does not correlate between tasks at the individual level. This asymmetry may be because of a benefit for rotating objects in the direction they are facing. An alternative would be that the front of left-facing objects is high on the screen for clockwise rotations and low for counterclockwise rotations, and vice versa for right-facing objects. Consistent with this notion, responses during top/bottom discriminations show RT benefits when the target dot is located high on the screen (Jolicoeur, Ingleton, Bartram, \& Booth, 1993; Light \& Hamm, 2008). Regardless of the underlying cause for the 
observed asymmetries, the lack of a relationship between an individual's asymmetry in performance between the tasks suggests that there may be multiple causes or that the cause may be more influenced by either the task or by the choice of stimuli. In contrast, the correlations between tasks of the other parameters suggest that these are measuring something common about the participant that transcends the different tasks, presumably common underlying mental processes.

As mentioned, although the asymmetries in the RT functions did not vary with task order, for those participants who performed the left/right facing task prior to the mirror/normal letter task, the orientation effect was smaller and the exponent value was larger for the mirror/normal letter task when compared with those participants who performed the mirror/normal letter task first. The corresponding values for the left/right facing task did not differ according to task order. This order effect suggests that performance of the left/right facing task transfers some practice benefits to the mirror/normal letter task. An increase in the exponent value indicates less reliance on mental rotation; therefore, both the reduction in the orientation effect and the increase in the exponent value could indicate that practice with the left/ right facing task enables participants to make mirror/normal letter judgements without resorting to mental rotation more often than those participants who do not have this practice. However, there is asymmetric transfer, since practice with the mirror/normal letter task does not seem to transfer to the left/right facing task.

The aforementioned transfer of practice benefits are easily explained with respect to the processes thought to be indexed by the orientation effect and mixture exponent values. Determining whether a letter is a mirror or normal requires polarizing the horizontal axis to determine whether the appropriate letter features are on the appropriate side. In Kung and Hamm's (2010) description of the nonrotation process, they suggested that knowledge of the intended direction of mental rotation enables this polarization of the horizontal axis to occur without the use of mental rotation. A plausible interpretation of the task order effect is that because the left/right facing task makes the spatial mapping between direction of rotation and left/right polarity explicitor at least emphasizes the relationship - this is suggested to benefit the nonrotation process. This increase in emphasis on the relationship may serve to reduce the spatial conflict, resulting in a reduced reliance on mental rotation, which is reflected in the increased exponent values. Performing the mirror/ normal letter task does not make this mapping as explicit, and therefore may be less prone to transfer.

Finally, using the data from three separate studies, it was possible to compare the results from published studies in which group data are reported. Because the value obtained for the mixture exponent fitted to group data is not the same as the average of the individual values, comparison with the literature required creating a distribution of group averages. The present data were compared with findings from Rogers et al. (2002), who reported that although nonmelancholic depressed individuals showed general slowing in their RTs relative to controls, they did not show a change in their mental rotation rates. In contrast, the melancholic depressed individuals showed both an overall slowing and an increase in the orientation effect, both relative to controls. This interpretation was also drawn by comparing the baseline RT and the orientation effect parameters derived from the presented data (Rogers et al., 2002), with a distribution of the parameters fitted to group data of the same size, which were randomly generated from a collection of 68 individuals. In addition, we were able to compare the mixture exponent values fitted to the published data and found that the mixture exponents for the control and nonmelancholic groups were not different from randomly generated groups, but that the mixture exponent for the melancholic individuals was smaller than expected. This indicates that although the melancholic patients appear to rotate more slowly, which could indicate that mental rotation is a more effortful process for them than for the other groups, they also appear to resort to rotation more frequently. This would suggest that the nonrotation process may be even more taxing on executive function - an interpretation consistent with a recent meta-analysis in which it was found that depression was negatively correlated with executive function rather than with visuospatial memory performance (McDermott \& Ebmeier, 2009). Holmes and Pizzagalli (2007) have also shown that higher Beck's Depression Inventory scores are associated with less conflict adaptation, meaning that this group did not show the RT benefits associated with incongruent trials that follow incongruent trials relative to incongruent trials that follow congruent trials. In the Holmes and Pizzagalli study, this finding was limited to the Simon task and did not replicate to the Stroop task, which they suggested may be related to the fact that the Simon task more strongly involves regions associated with response conflict, response selection, and planning. Given that spatial response conflict is proposed to be the trigger for mental rotation (Kung \& Hamm, 2010), this association with a spatial conflict task and depression warrants further investigation.

In summary, the addition of an exponent to the mixture ratio of Kung and Hamm's (2010) model of rotated mirror/ normal letter discriminations appears to be justified. The increase in model complexity is supported by the improved Akaike criterion score and also by the fact that the exponent value appears to correlate at the individual level between different tasks. Moreover, the value does not correlate with other parameters, such as the individual orientation effect, 
indicating that it is capturing different aspects of processing. The value of the exponent is thought to quantify the degree to which an individual responds without resorting to mental rotation. Being able to quantify individuals on this ability means that performance on the left/right and mirror/normal tasks needs to be interpreted with both mental rotation and this nonrotation process in mind. This may be especially useful when it comes to the examination of clinical populations. Furthermore, by examining behavior in terms of the model parameters, it was shown that practice on the left/ right facing task appears to provide benefits on the mirror/ normal letter task, but not vice versa. This asymmetric transfer of practice effects is also consistent with the recent proposal that it is spatial conflict that triggers mental rotation. And, finally, it should not be overlooked that the RT functions for both the mirror/normal letter task and the left/right facing task may each be influenced by as yet unexplained factors that produce asymmetries in the performance data. This characteristic of the data has yet to be adequately explained.

Author note We thank Evan F. Risko, Charles Leek, one anonymous reviewer, and the editor, David Waller, for their helpful comments and suggestions during the preparation of this manuscript. The research reported here was conducted in partial fulfilment for the requirements of the Bachelor of Science Honours Degree by J.A.S under the supervision of J.P.H. at The University of Auckland, Auckland, New Zealand. Correspondence concerning this work should be directed to J.P. Hamm (e-mail: j.hamm@auckland.ac.nz).

\section{Appendix A}

Table 2 Model parameters for all 24 participants for both the mirror/normal and left/right tasks, including the asymmetry values that are not accounted for by the current model

\begin{tabular}{|c|c|c|c|c|c|c|c|c|c|c|}
\hline \multicolumn{5}{|c|}{ Mirror/Normal Task } & \multicolumn{6}{|c|}{ Left/Right Task } \\
\hline \multirow[b]{2}{*}{ Participant } & \multicolumn{2}{|c|}{ Orientation } & \multicolumn{2}{|l|}{ Mixture } & \multicolumn{2}{|c|}{ Orientation } & \multicolumn{3}{|l|}{ Mixture } & \multirow{2}{*}{$\begin{array}{l}\mathrm{M} / \mathrm{N} \text { performed } \\
1 \text { st or } 2 \text { nd }\end{array}$} \\
\hline & Baseline & Effect & Exponent & Asymmetry & Baseline & Effect & Exponent & Asymmetry & Gender & \\
\hline 1 & 921 & 3.46 & 0.77 & 90.40 & 589 & 2.70 & 0.81 & 149.02 & $\mathrm{~m}$ & 1 \\
\hline 2 & 735 & 2.35 & 0.58 & 73.75 & 471 & 1.62 & 0.29 & 94.09 & $\mathrm{~m}$ & 1 \\
\hline 3 & 856 & 2.64 & 1.44 & 66.43 & 589 & 2.21 & 1.44 & 46.94 & $\mathrm{~m}$ & 2 \\
\hline 4 & 654 & 1.89 & 2.20 & -0.86 & 508 & 1.65 & 0.33 & 72.50 & $\mathrm{~m}$ & 2 \\
\hline 5 & 784 & 2.74 & 1.96 & 50.25 & 530 & 2.16 & 1.08 & 47.57 & $\mathrm{~m}$ & 1 \\
\hline 6 & 749 & 2.48 & 0.36 & 38.34 & 582 & 1.70 & 0.55 & 67.03 & $\mathrm{~m}$ & 1 \\
\hline 7 & 655 & 1.83 & 3.08 & 47.88 & 469 & 2.39 & 1.11 & 80.39 & $\mathrm{~m}$ & 2 \\
\hline 8 & 568 & 1.40 & 1.71 & 50.86 & 432 & 0.81 & 0.22 & 49.84 & $\mathrm{~m}$ & 2 \\
\hline 9 & 787 & 3.40 & 1.13 & -44.80 & 469 & 2.19 & 1.01 & 42.34 & $\mathrm{~m}$ & 1 \\
\hline 10 & 840 & 3.22 & 0.47 & -47.51 & 502 & 2.00 & 1.10 & 143.47 & $\mathrm{~m}$ & 1 \\
\hline 11 & 599 & 2.40 & 2.56 & 21.26 & 480 & 1.77 & 0.67 & 58.70 & $\mathrm{~m}$ & 2 \\
\hline 12 & 674 & 1.88 & 0.95 & 44.83 & 566 & 2.36 & 0.44 & 136.61 & $\mathrm{~m}$ & 2 \\
\hline 13 & 639 & 3.65 & 1.17 & 1.10 & 568 & 2.33 & 1.84 & 78.08 & $\mathrm{f}$ & 1 \\
\hline 14 & 563 & 2.51 & 1.19 & 58.32 & 453 & 2.53 & 1.26 & -21.91 & $\mathrm{f}$ & 1 \\
\hline 15 & 469 & 1.47 & 2.14 & 33.18 & 515 & 1.62 & 2.03 & 31.35 & $\mathrm{f}$ & 2 \\
\hline 16 & 736 & 6.13 & 1.19 & 77.46 & 579 & 2.55 & -0.08 & 281.18 & $\mathrm{f}$ & 2 \\
\hline 17 & 898 & 2.26 & 0.67 & 86.81 & 609 & 2.06 & 0.42 & 121.88 & $\mathrm{f}$ & 1 \\
\hline 18 & 911 & 2.95 & 0.95 & 20.77 & 537 & 2.82 & 0.44 & 96.91 & $\mathrm{f}$ & 1 \\
\hline 19 & 525 & 1.58 & 1.24 & 10.39 & 450 & 2.49 & 1.30 & 69.20 & f & 2 \\
\hline 20 & 575 & 1.93 & 1.82 & -6.56 & 510 & 3.13 & 1.67 & 27.42 & $\mathrm{f}$ & 2 \\
\hline 21 & 656 & 2.00 & 3.40 & 26.43 & 410 & 1.82 & 1.03 & 61.58 & f & 1 \\
\hline 22 & 829 & 2.92 & 0.95 & 51.95 & 621 & 1.62 & 0.04 & 108.53 & $\mathrm{f}$ & 1 \\
\hline 23 & 534 & 1.02 & 2.26 & 17.56 & 448 & 1.99 & 1.45 & 66.55 & $\mathrm{f}$ & 2 \\
\hline 24 & 742 & 2.25 & 3.18 & 40.20 & 499 & 2.62 & 1.47 & 75.11 & $\mathrm{f}$ & 2 \\
\hline
\end{tabular}


Fig. 4 Observed and modeled response time data in milliseconds for Participants 1 through 6 for the mirror/normal task. A and B codes beside the participant numbers indicate task order a mirror/normal followed by left/right; b left/right followed by mirror/normal
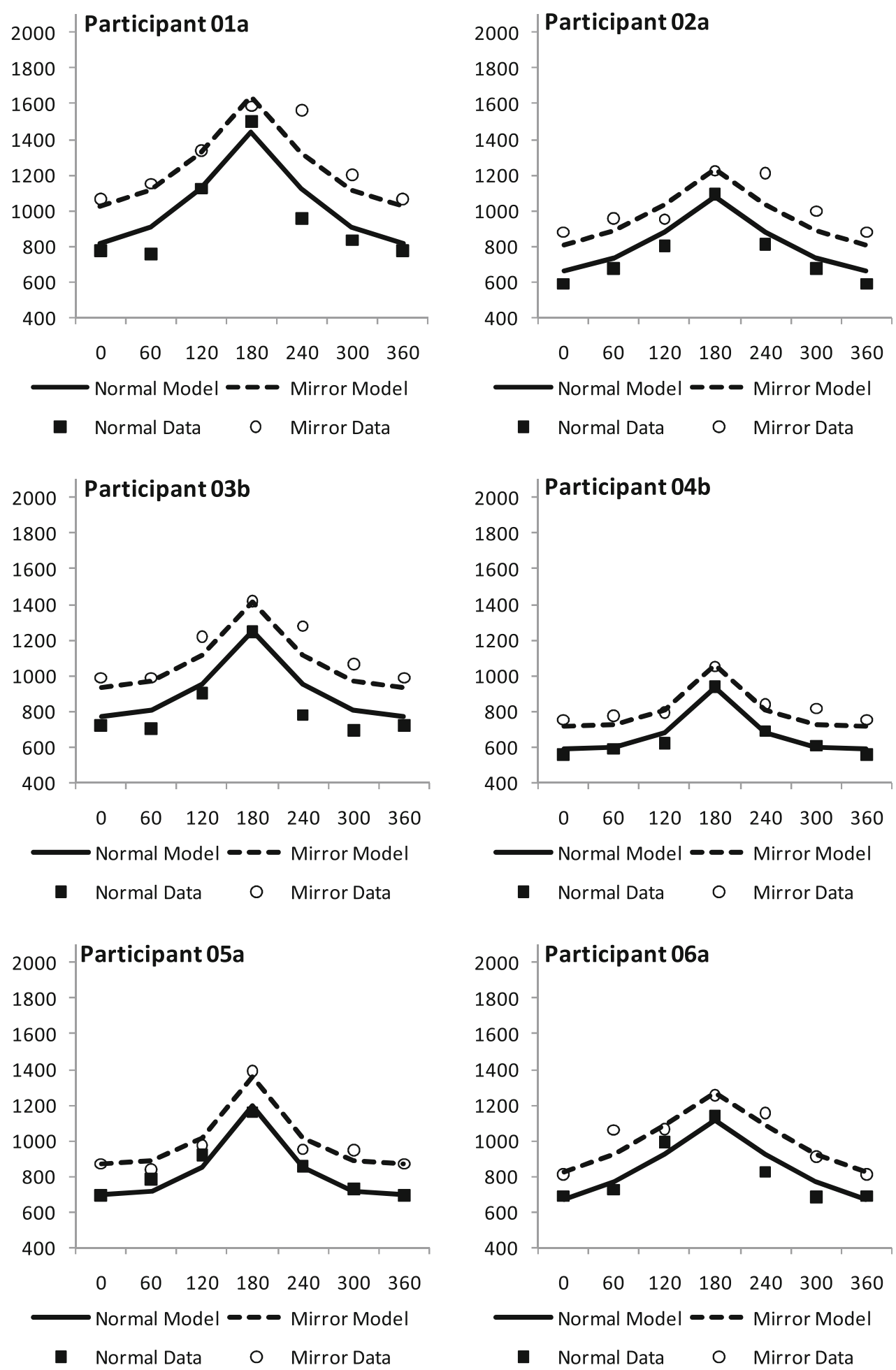
Fig. 5 Observed and

modeled response time data in milliseconds for Participants

7 through 12 for the

mirror/normal task. A and B

codes beside the participant

numbers indicate task order

a mirror/normal followed by

left/right; b left/right followed

by mirror/normal
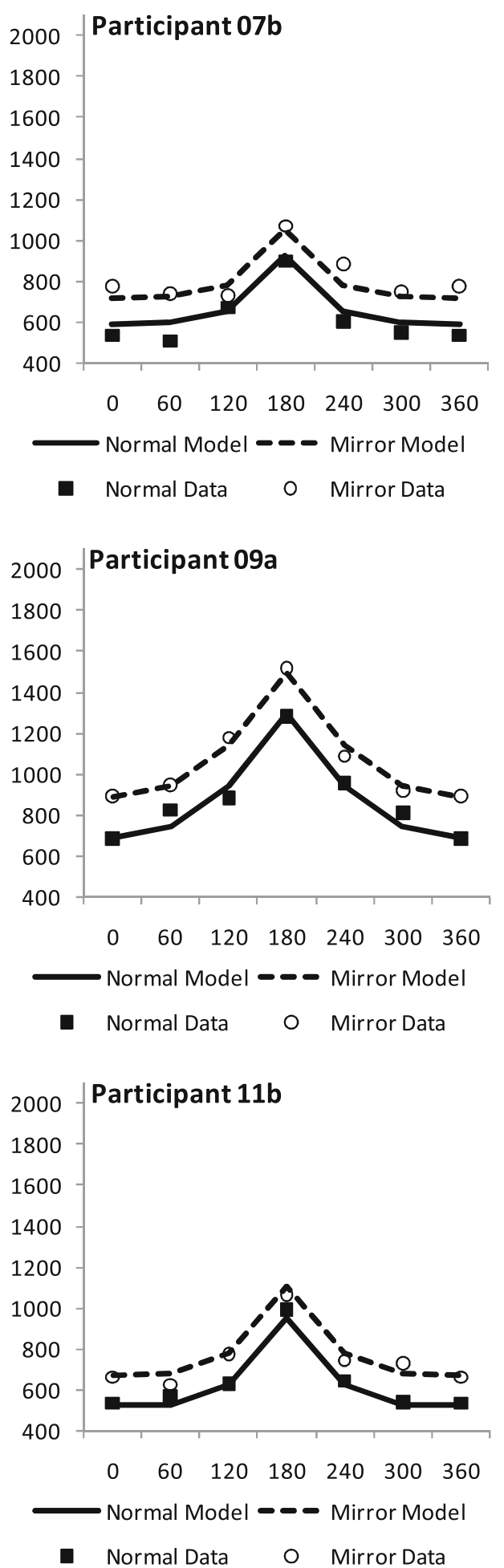
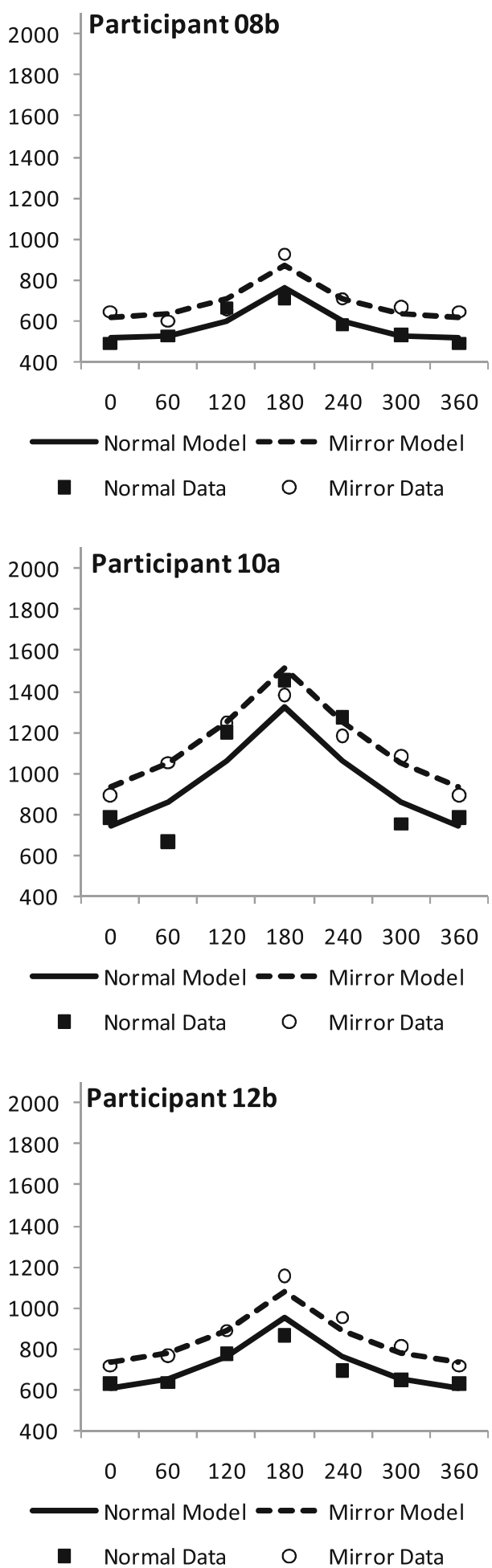
Fig. 6 Observed and modeled response time data in milliseconds for Participants 13 through 18 for the mirror/normal task. A and B codes beside the participant numbers indicate task order a mirror/normal followed by left/right; b left/right followed by mirror/normal
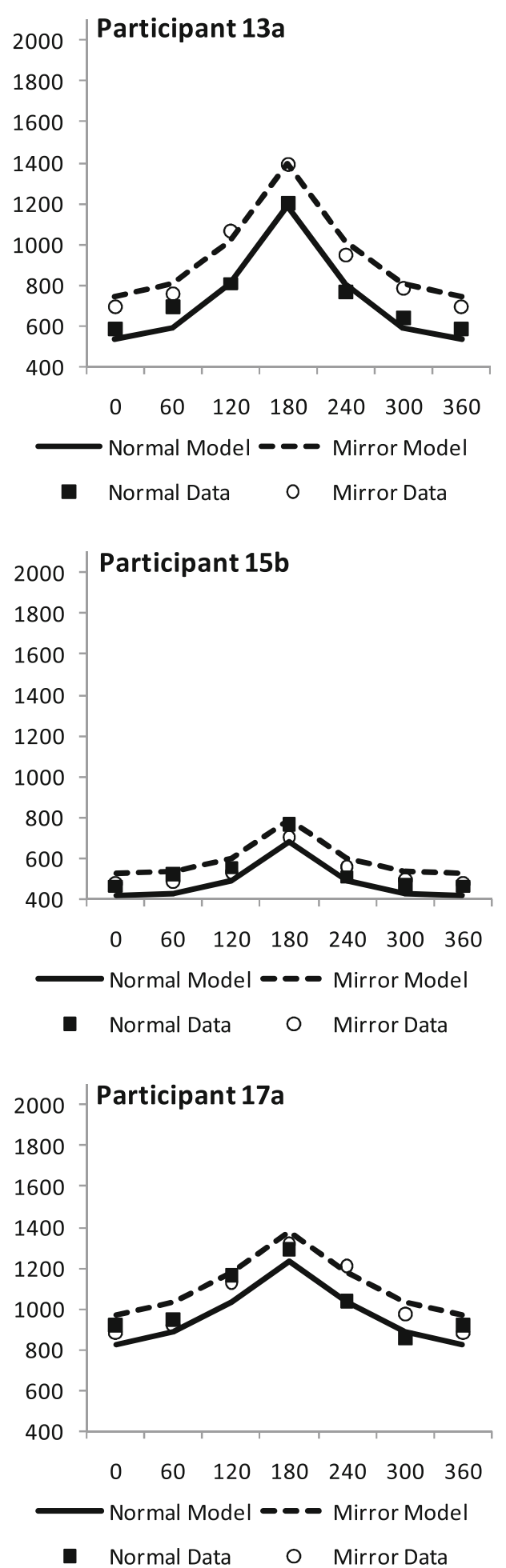
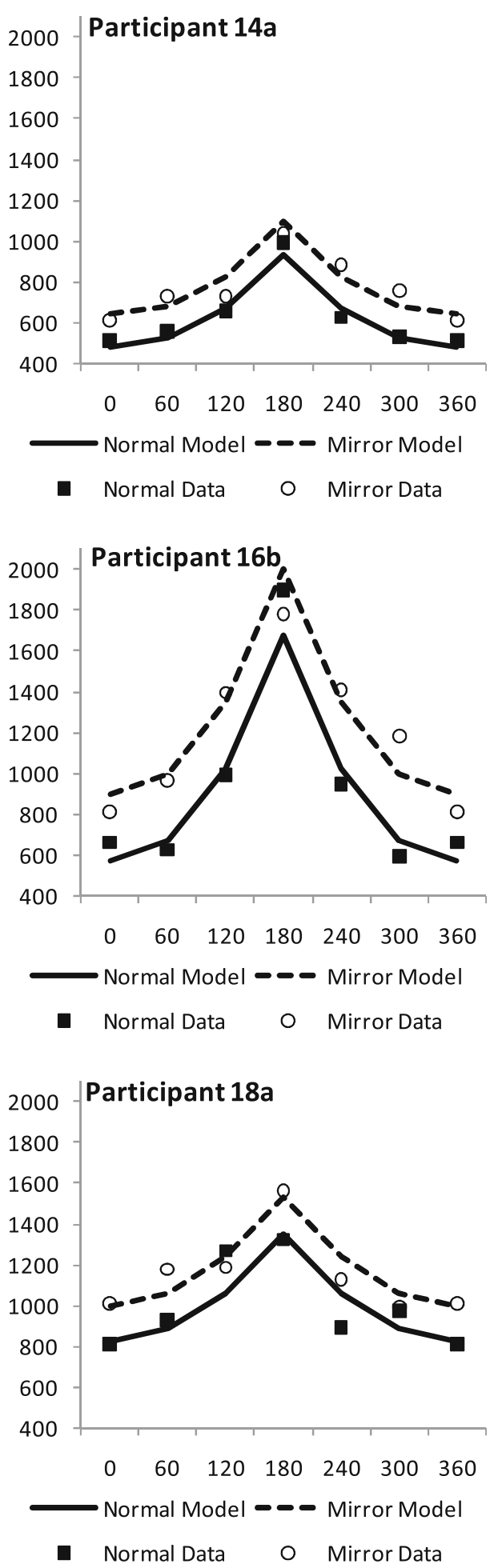
Fig. 7 Observed and modeled response time data in milliseconds for Participants 19 through 24 for the mirror/normal task. A and B codes beside the participant numbers indicate task order a mirror/normal followed by left/right; b left/right followed by mirror/normal
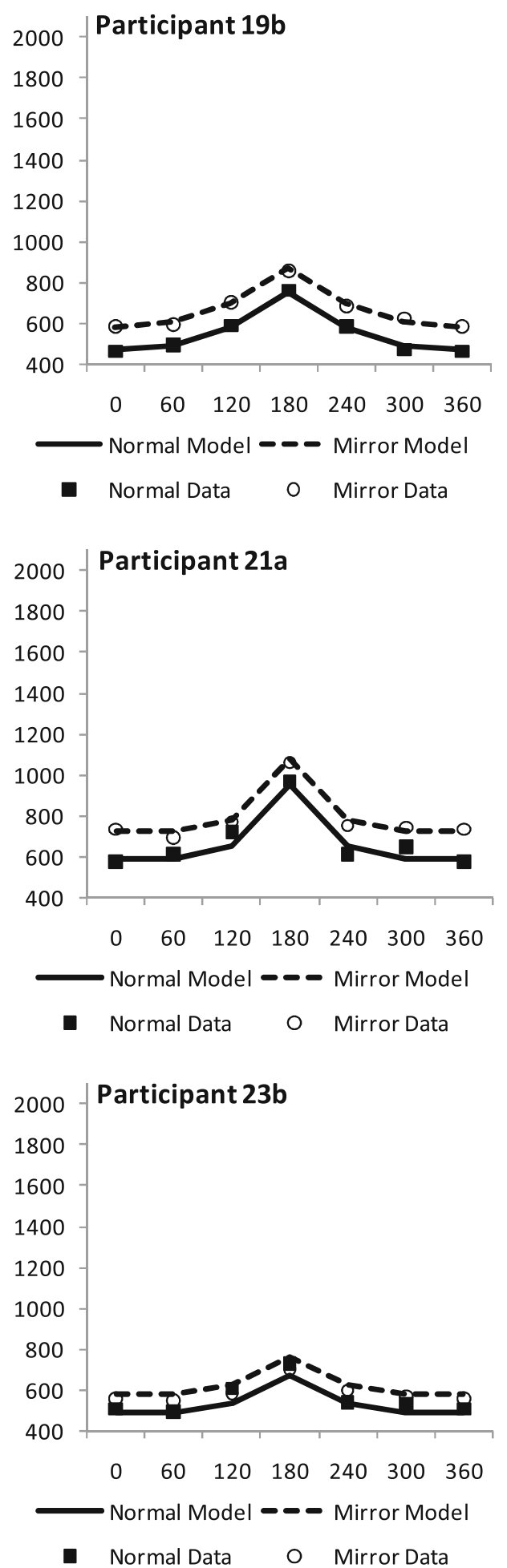
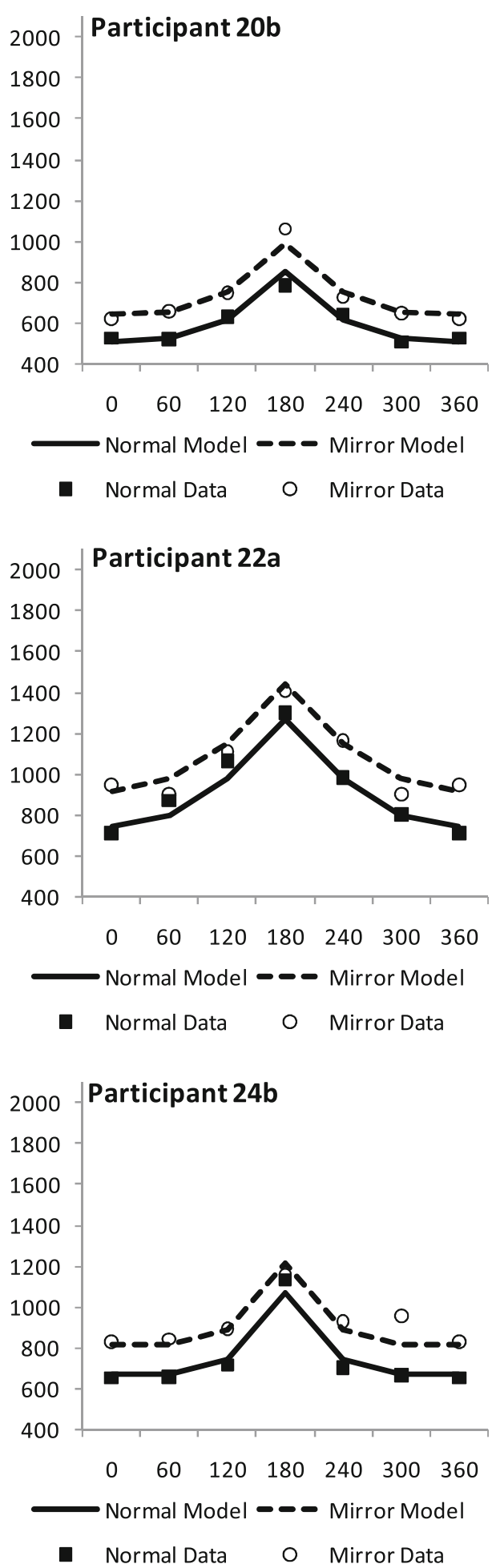


\section{Appendix B}

Fig. 8 Observed and modeled response time data in milliseconds for Participants 1 through 6 for the left/right task. A and B codes beside the participant numbers indicate task order a mirror/normal followed by left/right; b left/right followed by mirror/normal
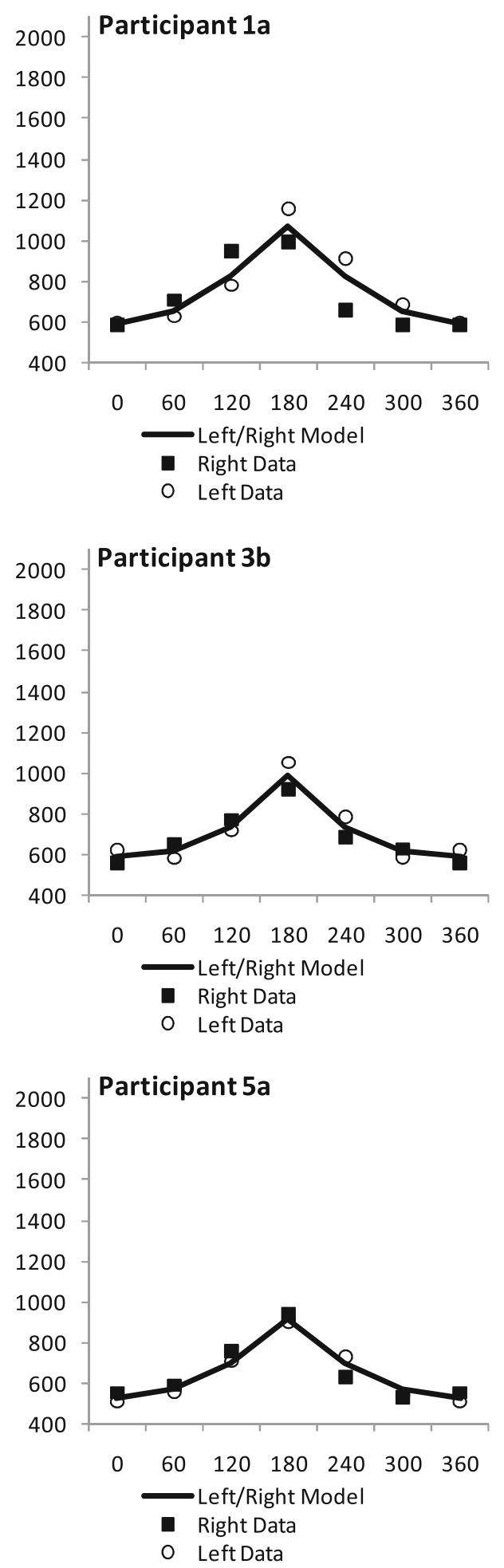
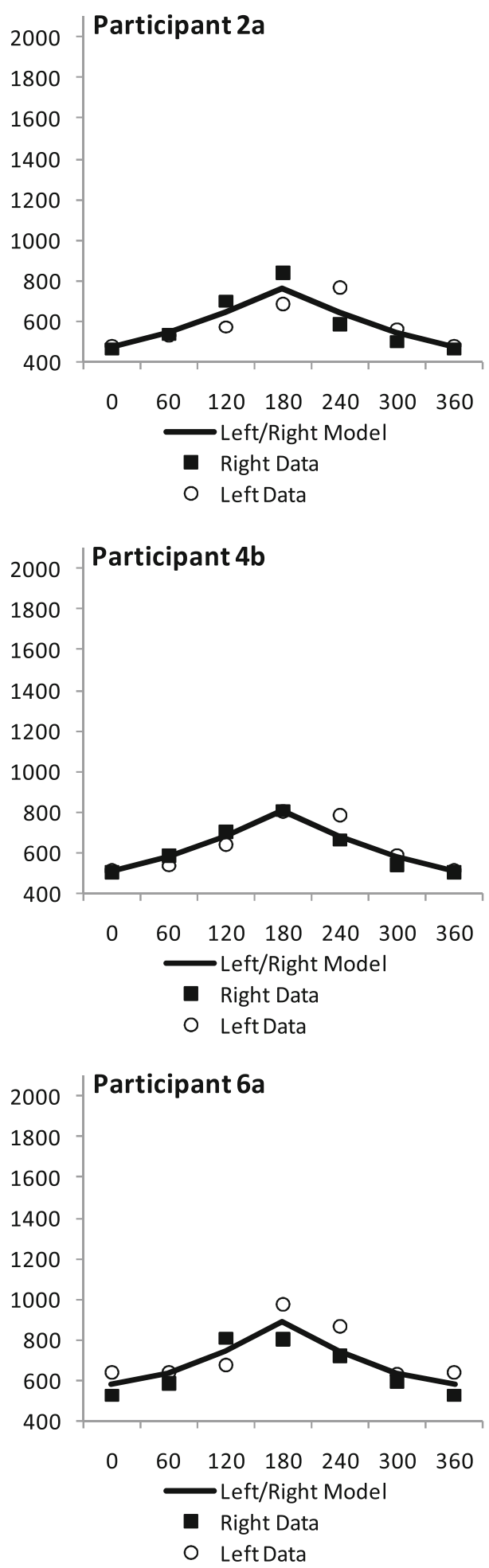
Fig. 9 Observed and modeled response time data in milliseconds for Participants 7 through 12 for the left/right task. A and B codes beside the participant numbers indicate task order a mirror/normal followed by left/right; b left/right followed by mirror/normal
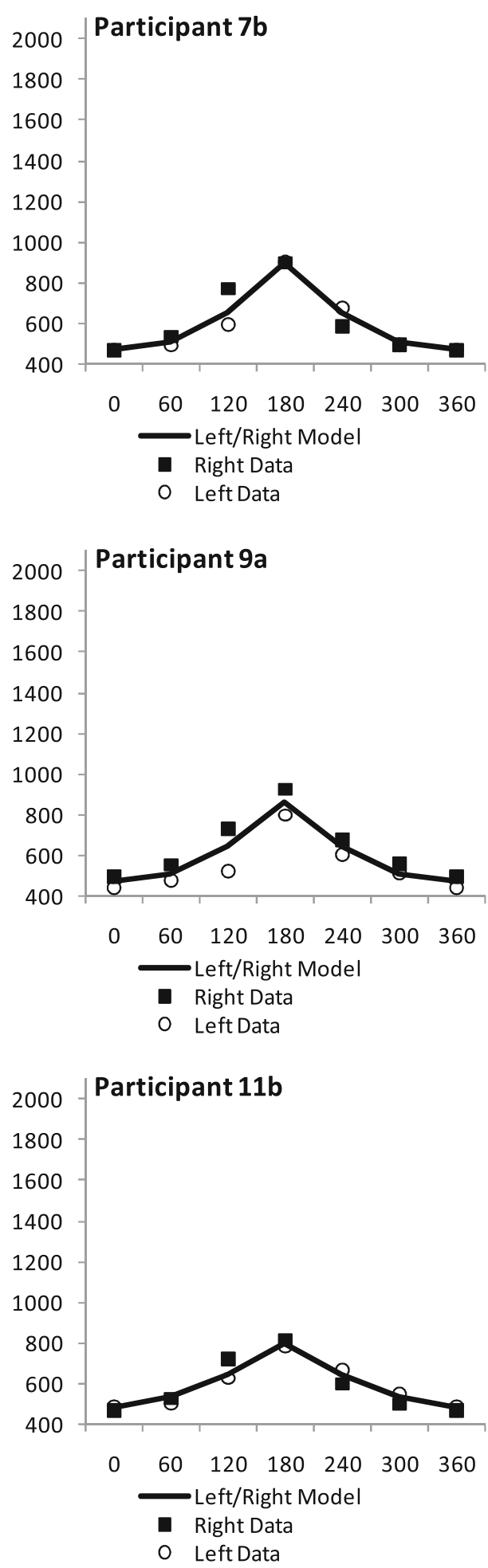
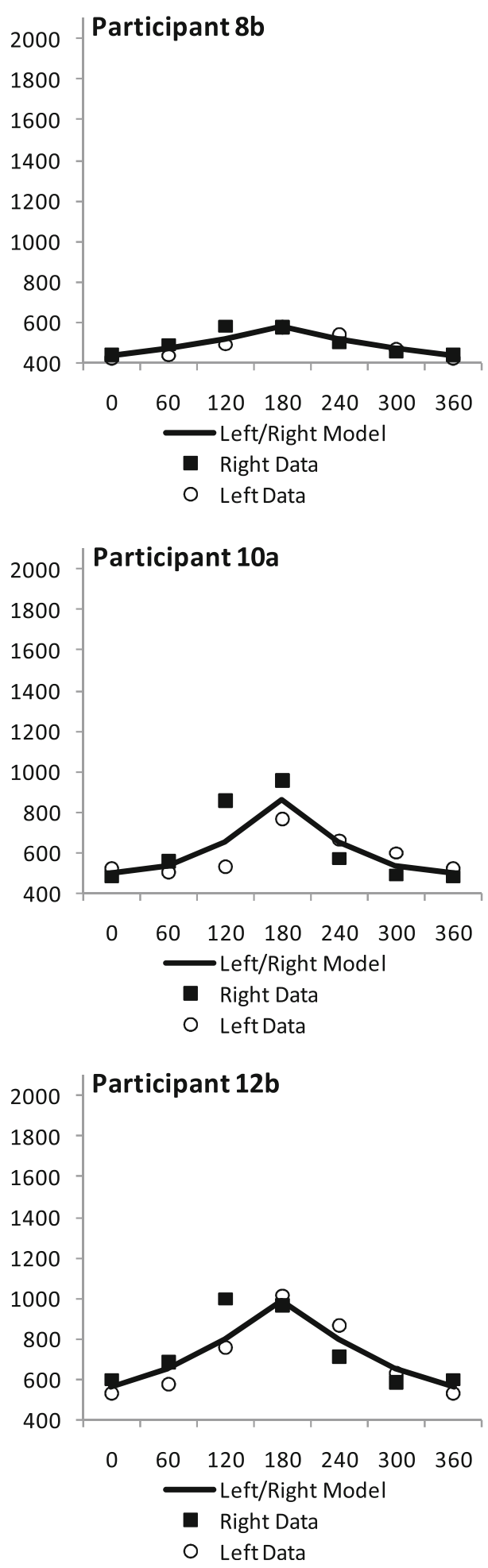
Fig. 10 Observed and modeled response time data in milliseconds for Participants 13 through 18 for the left/right task. A and B codes beside the participant numbers indicate task order a mirror/normal followed by left/right; b left/right followed by mirror/normal
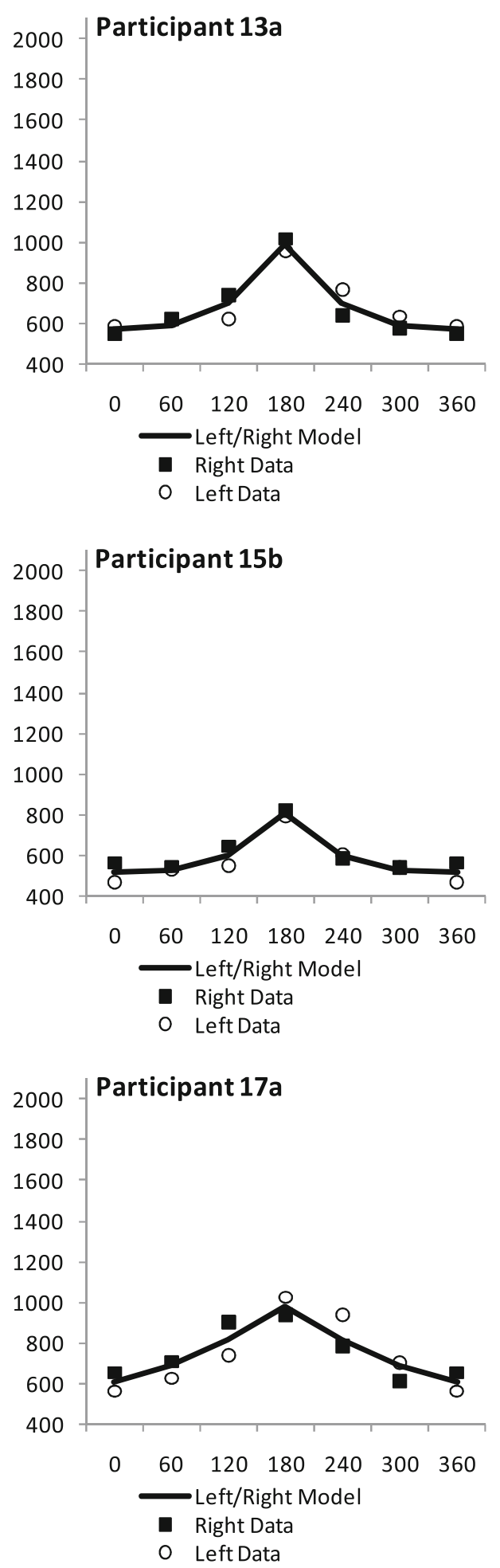
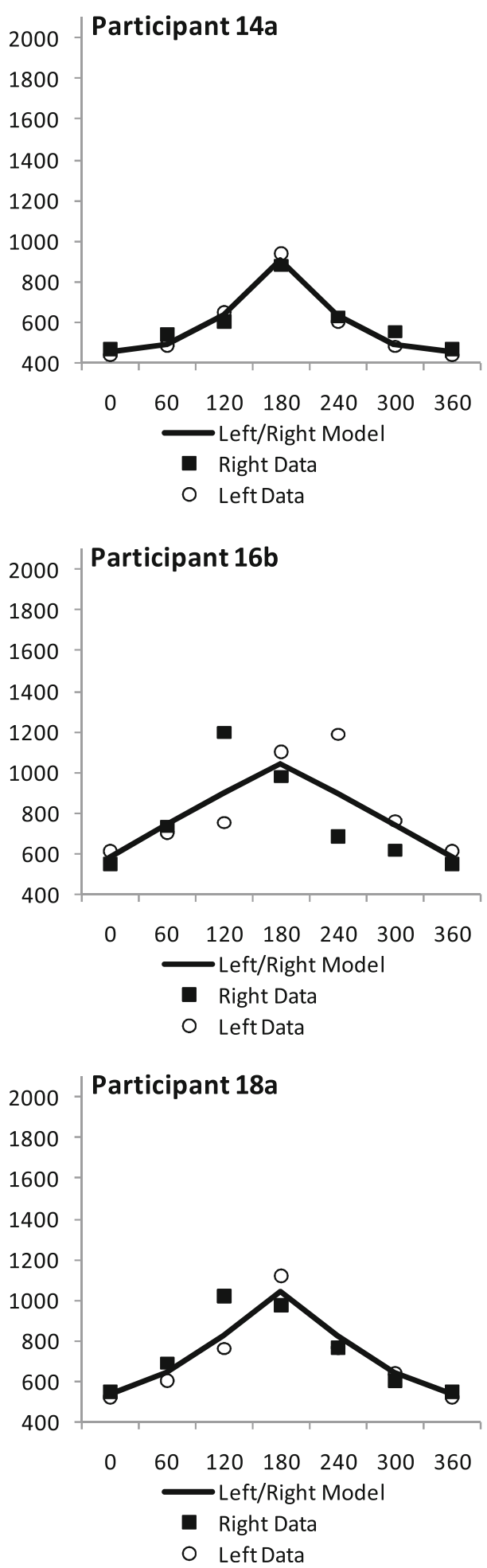
Fig. 11 Observed and modeled response time data in milliseconds for Participants 19 through 24 for the left/right task. A and B codes beside the participant numbers indicate task order a mirror/normal followed by left/right; b left/right followed by mirror/normal
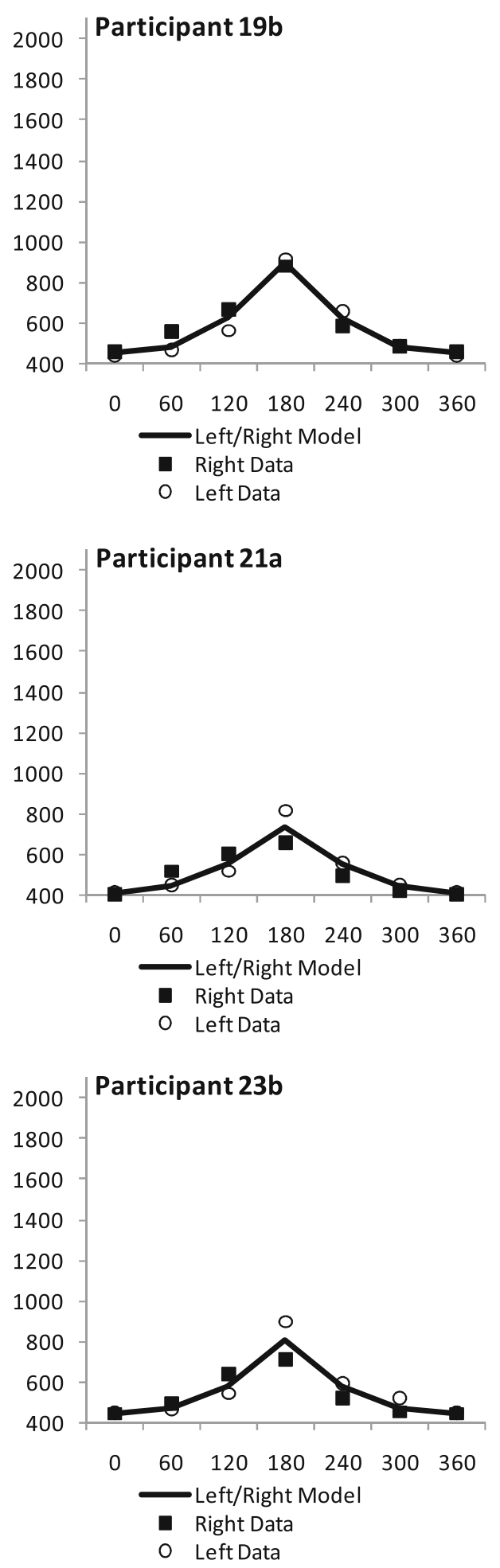
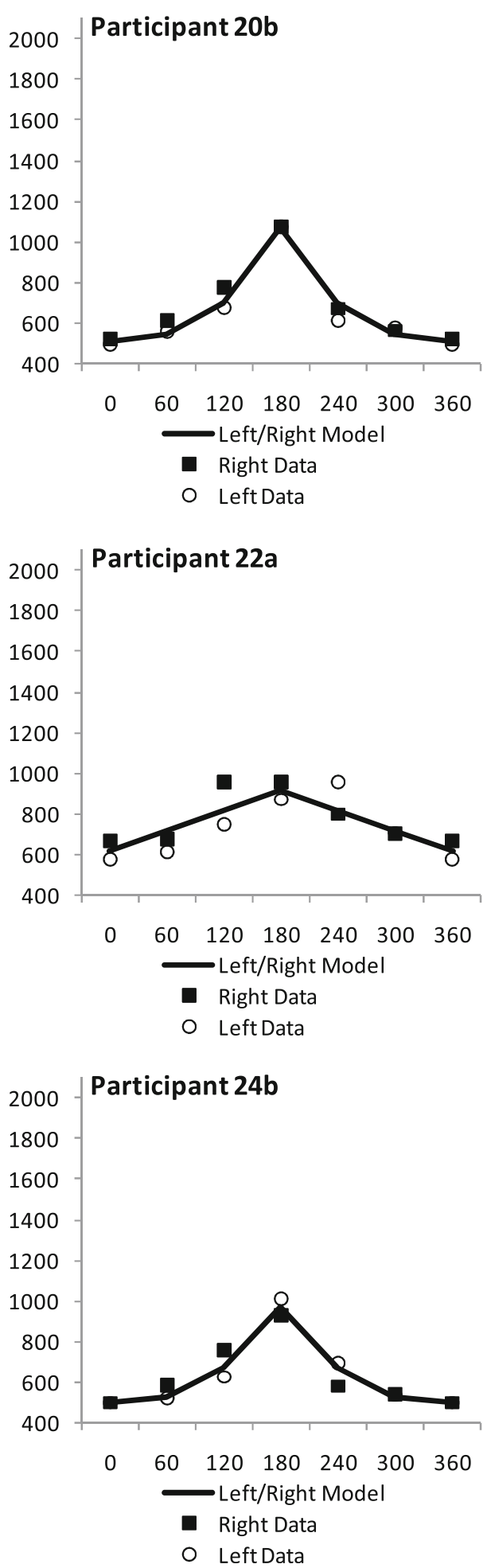


\section{References}

Amick, M. M., Schendan, H. E., Ganis, G., \& Cronin-Golomb, A. (2006). Frontostriatal circuits are necessary for visuomotor transformation: Mental rotation in Parkinson's disease. Neuropsychologia, 44, 339-349.

Burnham, K. P., \& Anderson, D. R. (2002). Model selection and multi-model inference: A practical information-theoretic approach (2nd ed.). New York, NY: Springer-Verlag.

Cooper, L. A., \& Shepard, R. N. (1973). Chronometric studies of the rotation of mental images. In W. G. Chase (Ed.), Visual information processing (pp. 75-176). New York, NY: Academic Press.

Corballis, M. C. (1982). Mental rotation: Anatomy of a paradigm. In M. Potegal (Ed.), Spatial abilities: Developmental and physiolgical foundations (pp. 75-176). New York, NY: Academic Press.

Corballis, M. C. (1988). Recognition of disoriented shapes. Psychological Review, 95, 115-123.

Corballis, M. C., \& McLaren, R. (1984). Winding one's Ps and Qs: Mental rotation and mirror-image discrimination. Journal of Experimental Psychology. Human Perception and Performance, 10, 318-327.

De Caro, S. A., \& Reeves, A. (2000). Rotating objects to determine orientation, not identity: Evidence from a backward-masking/dualtask procedure. Perception \& Psychophysics, 62, 1356-1366.

Hamm, J. P. (2001). Object-oriented millisecond timers for the PC. Behavior Research Methods, Instruments, \& Computers, 33, 532-539.

Hamm, J. P., Johnson, B. W., \& Corballis, M. C. (2004). One good turn deserves another: An event-related brain potential study of rotated mirror-normal letter discriminations. Neuropsychologia, 42, 810-820.

Hamm, J. P., \& McMullen, P. A. (1998). Effects of orientation on the identification of rotated objects depend on the level of identity. Journal of Experimental Psychology. Human Perception and Performance, 24, 413-426.

Harris, I. M., Harris, J. A., \& Caine, D. (2002). Mental-rotation deficits following damage to the right basal ganglia. Neuropsychology, 16, 524-537.

Heathcote, A. (1988). Screen control and timing routines for the IBM microcomputer family using a high-level language. Behavior Research Methods, Instruments, \& Computers, 20, 289-297.
Holmes, A. J., \& Pizzagalli, D. A. (2007). Task feedback effects on conflict monitoring and executive control: Relationship to subclinical measures of depression. Emotion, 7, 68-76.

Jolicoeur, P. (1988). Mental rotation and the identification of disoriented objects. Canadian Journal of Psychology, 42, 239-243.

Jolicoeur, P. (1990). Identification of disoriented objects: A dualsystems theory. Mind \& Language, 5, 387-410.

Jolicoeur, P., Ingleton, M., Bartram, L., \& Booth, K. S. (1993). Topbottom and front-behind decisions on rotated objects. Canadian Journal of Experimental Psychology, 47, 657-677.

Kung, E., \& Hamm, J. P. (2010). A model of rotated mirror/normal letter discriminations. Memory \& Cognition, 38, 206-220.

Light, P., \& Hamm, J. P. (2008). Stimulus response compatibilities during top bottom discriminations. Canadian Journal of Experimental Psychology, 62, 81-90.

McDermott, L. M., \& Ebmeier, K. P. (2009). A meta-analysis of depression severity and cognitive function. Journal of Affective Disorders, 119, 1-8.

McMullen, P. A., \& Jolicoeur, P. (1990). The spatial frame of reference in object naming and discrimination of left-right reflections. Memory \& Cognition, 18, 99-115.

Oldfield, R. C. (1971). The assessment and analysis of handedness: The Edinburgh Inventory. Neuropsychologia, 9, 97-113.

Parsons, L. M. (1987). Imagined spatial transformations of ones hands and feet. Cognitive Psychology, 19, 178-241.

Rogers, M. A., Bradshaw, J. L., Phillips, J. G., Chiu, E., Mileshkin, C., \& Vaddadi, K. (2002). Mental rotation in unipolar major depression. Journal of Clinical and Experimental Neuropsychology, 24, 101-106.

Shepard, R. N., \& Metzler, J. (1971). Mental rotation of threedimensional objects. Science, 171, 701-703.

Thayer, Z. C., \& Johnson, B. W. (2006). Cerebral processes during visuo-motor imagery of hands. Psychophysiology, 43, 401-412.

Thayer, Z. C., Johnson, B. W., Corballis, M. C., \& Hamm, J. P. (2001), Perceptual and motor mechanisms for mental rotation of human hands. NeuroReport, 12, 3433-3437.

Wagenmakers, E. J., \& Farrell, S. (2004). AIC model selection using Akaike weights. Psychonomic Bulletin \& Review, 11, 192-196. 